\title{
Reduced myelin in patients with isolated hippocampal sclerosis as assessed by SyMRI
}

\author{
Safak Parlak ${ }^{1}$ (1) - Gokcen Coban ${ }^{1} \cdot$ Ekim Gumeler $^{1} \cdot$ Jale Karakaya $^{2} \cdot$ Figen Soylemezoglu $^{3} \cdot$ Irsel Tezer $^{4}$. \\ Burcak Bilginer $^{5} \cdot$ Serap Saygi ${ }^{4} \cdot$ Kader K. Oguz $^{1}$
}

Received: 12 August 2021 / Accepted: 25 September 2021 / Published online: 5 October 2021

(c) The Author(s), under exclusive licence to Springer-Verlag GmbH Germany, part of Springer Nature 2021

\begin{abstract}
Purpose Synthetic MRI (SyMRI) enables to quantify brain tissue and morphometry. We aimed to investigate the WM and myelin alterations in patients with unilateral hippocampal sclerosis (HS) with SyMRI.

Methods Adult patients with isolated unilateral HS and age-matched control subjects (CSs) were included in this study. The SyMRI sequence QRAPMASTER in the coronal plane perpendicular to the hippocampi was obtained from the whole brain. Automatic segmentation of the whole brain was processed by SyMRI Diagnostic software (Version 11.2). Two neuroradiologists also performed quantitative analyses independently from symmetrical 14 ROIs placed in temporal and extratemporal WM, hippocampi, and amygdalae in both hemispheres.

Results Sixteen patients $(\mathrm{F} / \mathrm{M}=6 / 10$, mean age $=32.5 \pm 11.3$ years; right/left HS: $8 / 8)$ and $10 \mathrm{CSs}(\mathrm{F} / \mathrm{M}=5 / 5$, mean age $=30.7 \pm 7$ years) were included. Left HS patients had significantly lower myelin and WM volumes than CSs $(p<.05)$. Myelin was reduced significantly in the ipsilateral temporal lobe of patients than CSs, greater in left HS $(p<.05)$. Histopathological examination including luxol fast blue stain also revealed myelin pallor in all of 6 patients who were operated. Ipsilateral temporal pole and sub-insular WM had significantly reduced myelin than the corresponding contralateral regions in patients $(p<.05)$. No significant difference was found in WM values. GM values were significantly lower in hippocampi in patients than CSs $(p<.05)$.

Conclusion SyMRI revealed myelin reduction in the ipsilateral temporal lobe and sub-insular WM of patients with HS. Whether this finding correlates with electrophysiological features and SyMRI could serve as lateralization of temporal lobe epilepsy need to be investigated.
\end{abstract}

Keywords Synthetic MRI · Hippocampal sclerosis $\cdot$ Myelin $\cdot$ Epilepsy

\begin{tabular}{|c|c|c|c|}
\hline & & Abbrevia & \\
\hline & & BPV & Brain parenchymal volume \\
\hline & & $\mathrm{BPF}$ & Brain parenchymal fraction (BPV/ICV) \\
\hline & art of this study was presented in RSNA 2020 as a poster & $\mathrm{CS}$ & Control subject \\
\hline pre & sentation. & FA & Fractional anisotropy \\
\hline & $\begin{array}{l}\text { Safak Parlak } \\
\text { parlaksafak@gmail.com }\end{array}$ & GMFvol & $\begin{array}{l}\text { The mean amount of GM within a } \\
\text { single voxel }\end{array}$ \\
\hline & & HS & Hippocampal sclerosis \\
\hline 1 & Department of Radiology, Hacettepe University Faculty & $\mathrm{ICC}$ & Intra-class correlation coefficient \\
\hline & of Medicine, Ankara, Turkey & $\mathrm{ICV}$ & Intracranial volume \\
\hline 2 & Department of Biostatistics, Hacettepe University Faculty & MD & Mean diffusivity \\
\hline & of Medicine, Ankara, Iurkey & MY & Myelin \\
\hline 3 & Department of Pathology, Hacettepe University Faculty & MYF & Myelin fraction (MY/BPV) \\
\hline & of Medicine, Ankara, Iurkey & MyFvol & Myelin-correlated fraction volume \\
\hline 4 & Department of Neurology, Hacettepe University Faculty & & within a single voxel \\
\hline & 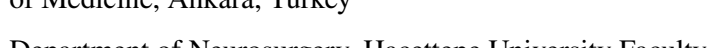 & $\mathrm{MyV}$ & Myelin-correlated volume \\
\hline & $\begin{array}{l}\text { Department of Neurosurgery, Hacettepe University Faculty } \\
\text { of Medicine, Ankara, Turkey }\end{array}$ & $\mathrm{NoN}$ & Non-GM/WM/CSF \\
\hline
\end{tabular}


PD

\section{QRAPMASTER}

Proton density

Quantification of relaxation times and proton density by multi-echo acquisition of a saturation-recovery using turbo spin-echo readout

R1

$\mathrm{R} 2$

SyMRI

TLE

WMFvol
Longitudinal relaxation rate

Transverse relaxation rate

Synthetic MRI (synthesized)

Temporal lobe epilepsy

The mean amount of WM within a single voxel

\section{Introduction}

Hippocampal sclerosis (HS) is the most common cause of refractory temporal lobe epilepsy (TLE) in adults [1] and consists of neuronal loss with glial tissue proliferation histopathologically. Magnetic resonance imaging (MRI) has a sensitivity of $91 \%$ for detecting focal epileptogenic lesions [2] and 93\% and $83 \%$ for detecting hippocampal and amygdala abnormalities, respectively [3]. The imaging findings of HS on MRI include the following: increased T2/FLAIR signal intensity in the hippocampus, loss of internal structure of the hippocampus, reduced hippocampal size and related ipsilateral atrophy of the hippocampal collateral WM, enlarged temporal horn of lateral ventricle, diminished gray-white matter demarcation in the temporal pole, and decreased temporal lobe volume [3-6]. Atrophy of the ipsilateral mammillary body, fornix, and thalamus can also be seen [7]. Ipsilateral temporal pole signal abnormality in HS is another frequently associated finding on MRI $[8,9]$.

Synthetic MRI (SyMRI) is an FDA-approved unique faster MRI technique that is a compilation pulse sequence (multi-slice, multi-echo, multi-saturation delay acquisition) and enables the creation of multiple sequences based on the relaxation time of the tissue properties from a single acquisition. It can also automatically create intracranial tissue segmentation [10]. SyMRI was previously examined in several studies in patients with cognitive impairment [11], SturgeWeber [12], multiple sclerosis [13-15], and brain metastases [16-18]. Diagnostic software Version 11.2 of SyMRI also allows quantification of WM, GM, and myelin-correlated volume (MyV) in the ROIs (https://syntheticmr.com/produ cts/symri-neuro/).

We hypothesized that SyMRI quantitatively would reveal myelin alterations in temporal and extratemporal structures of patients with unilateral TLE-HS.

\section{Methods}

This study was approved by our institutional review board and informed patient consents were obtained.

\section{Patient selection}

Patients diagnosed with HS between November 2019 and December 2020 were enrolled in the study prospectively $(n=20)$. All patients had brain MRIs applying epilepsydedicated protocol including T2W imaging obtained perpendicular to the hippocampi. These scans were reviewed, and the final diagnosis was confirmed independently by two neuroradiologists (KKO with 20 and GC with 8 years experience in epilepsy imaging).

The patients were invited to participate in the study if they met the inclusion criteria which were (i) clinical diagnosis and electroencephalography (EEG) findings supporting TLE, (ii) age $>18$ years, and (iii) MRI demonstrating unilateral HS (increased T2/FLAIR signal intensity in the hippocampus, loss of internal structure of the hippocampus, reduced hippocampal size). Exclusion criteria were (i) bilateral HS on MRI, (ii) presence of any other structural lesion, and (iii) history of seizure within 1 week.

A total of 16 adult patients with unilateral isolated HS were included in this study. Control subjects (CSs) were chosen from age-matched patients who were referred to our department with headache, vertigo, or peripheral facial paralysis $(n=10)$ with normal brain MRI findings.

\section{MR image acquisition}

All MR scans were acquired with a $1.5 \mathrm{~T}$ scanner (Magnetom, Aera, Siemens), equipped with a 20-channel standard head coil. Coronal T2W TSE (FOV: $199 \times 220 \mathrm{~mm}$; TR/TE: 6560/95 ms; FA: 150 ; ST: $3 \mathrm{~mm}$; NEX: 3 ) and FLAIR (FOV: $186 \times 230 \mathrm{~mm}$; TR/TE/TI: 7000/78/2229 ms; FA: $180^{\circ}$; ST: $4 \mathrm{~mm}$; NEX: 1) were added as conventional MRI to use as reference images for visual assessment. The SyMRI sequence (QRAPMASTER: quantification of relaxation times and proton density by multi-echo acquisition of a saturation-recovery using turbo spin-echo readout) (FOV: $230 \times 230 \mathrm{~mm}$; voxel size: $1.5 \times 1.5 \mathrm{~mm}$; TE/ TR/TI: $14,28,42,56,70 \mathrm{~ms} / 7000 / 0.0974,0.5846,1.8511$, $4.0919 \mathrm{~ms}$; FA: $150^{\circ}$; ST: $4 \mathrm{~mm}$, slice gap $=1 \mathrm{~mm}$, acquisition time $=6.05 \mathrm{~min}$ ) was obtained covering the whole brain on the coronal plane perpendicular to the hippocampi. No contrast material was used.

\section{SyMRI technique}

SyMRI (Synthetic MR AB, Linköping, Sweden) enables variable contrast-weighted images after quantifying the longitudinal relaxation rate $(\mathrm{R} 1)$, the transverse relaxation rate 
(R2), and the proton density (PD) and can create intracranial volume (ICV), GM, WM, CSF, non-GM/WM/CSF (NoN), myelin (MY), and myelin fraction volume (MYF) quantification of the whole brain [10].

The myelin, free water, cellular, and excess parenchymal water contribute to the MRI acquisition voxel [19]. Because each compartment has different relaxation properties, myelin volume can be estimated. The total volume of myelin in addition to WM, GM, CSF, and NoN can be calculated by multiplying the summed volume fractions for each tissue type based on predefined tissue characteristics. Among these, NoN represents the tissue not classified as GM, WM, and CSF [19-21].

Colored maps of WM, GM, CSF, and MyV can be automatically created, and the MyV map was demonstrated to have a high correlation with the partial volume of myelin in each voxel (https://syntheticmr.com/products/symri-neuro/). Normal appearing myelin-correlated compound on $\mathrm{MyV}$ map appears bright fluorescent yellow and green colors (yellow > green) (https://syntheticmr.com/wp/custom/uploads/ 2021/02/SyMRI-NEURO.pdf). Normal GM and WM are coded as green and pink colors, respectively.

Quantitative values consisting of R1, R2, PD, GM, WM, $\mathrm{MyV}$, and volume (mean amount of a single voxel) of GM (GMFvol), WM (WMFvol), and MyV (MyFvol), were also automatically provided for selected ROIs by Diagnostic software Version 11.2.

\section{Image analysis}

The QRAPMASTER sequences were evaluated to identify artifacts before uploading to the software. Whole brain automatic segmentation yielded MY, BPV, BPF, ICV, MYF, $\mathrm{GM}$, and nonWM/GM/CSF volumes in all subjects. The colored myelin, GM, and WM maps were displayed. The segmented tissue properties were overlaid on the synthetic T1W sequence.

The quantitative analyses of myelin, WM, and GM maps were performed independently and individually by two neuroradiologists (SP and GC).

Region of interest (ROI) sizes ranged as follows allowing a sensitive sampling depending on the region: (1) bilateral temporal poles, temporal stem, posterior temporal and subinsular region, and corpus callosum (genu, corpus, splenium) (20-22 ml); (2) superior and inferior frontal, parietal, and occipital WM and on the thalamus, on the myelin, and WM maps (40-42 ml) (Supplementary illustrated Fig. 1); and (3) bilateral hippocampi and amygdalae on the GM maps (20-22 ml).

The MyV, MyFvol, WM, WMFvol, GM, GMFvol, R1, and $\mathrm{R} 2$ relaxation rates and $\mathrm{PD}$ values were noted for each measurement. With the mirror copy option available in this version, ROIs drawn on an image set were copied to the contralateral side. The program also allowed that when an ROI was drawn on a single set, the other imaging sets also displayed the same ROI. These features let us avoid any misalignment while collecting data from different hemispheres or different maps.

\section{Clinical evaluation}

The age of onset and duration of epilepsy, presence of predisposing or risk factors for epilepsy (history of febrile convulsion, meningitis/encephalitis, trauma, or perinatal brain injury), and presence of secondary generalized seizures or status epilepticus were recorded.

\section{Histopathological evaluation}

To observe myelin under light microscopy, luxol fast blue stain was added to routinely performed H\&E and immunohistochemical stains. Histopathologic evaluation was done by a neuropathologist (FS) with an experience of $>30$ years. The reduction in myelin was assessed visually by luxol fast blue (LFB) staining in comparison with autopsy sections obtained from patients without neurological disease. The evaluation was performed in a blinded fashion.

\section{Statistical analysis}

Quantitative variables were evaluated with the Kolmogorov-Smirnov test to reveal the presence of normal distribution. Descriptive statistics were expressed as mean \pm standard deviation if variables were normally distributed or median (min-max) if variables did not show normal distribution. Independent sample $t$-test was used to compare normally distributed variables; otherwise, the Mann-Whitney $U$ test was used. Paired sample $t$-test was used to compare the myelin amount of the different hemispheres in HS patients. If the data did not meet the normal distribution condition, two paired sample comparisons were performed using the Wilcoxon test. Comparison of left- and right-sided HS subgroups with side-matched hemispheres of CSs was assessed by independent sample $t$-test or Mann-Whitney $U$ test. The relationship between quantitative values and clinical features was examined with the Pearson correlation or Spearman Rho correlation coefficient.

For comparisons of continuous variables, the adjusted $p$ value was obtained using the Benjamini-Hochberg method.

For the assessment of inter-observer agreement, intraclass correlation coefficient (ICC) was calculated. $p<0.05$ was used as a reference setpoint for statistical significance. 
IBM SPSS 21.0 Windows program was used for all statistical analyses.

\section{Results}

There was no statistical significance in terms of gen$\operatorname{der}(p=0.689)$ and age $(p=0.384)$ between the patients $(\mathrm{F} / \mathrm{M}=6 / 10$, mean age $=32.5 \pm 11.3$ (range $19-48)$ years) and $\mathrm{CSs}(\mathrm{F} / \mathrm{M}=5 / 5$, mean age $=30.7 \pm 7$ years (range 26-40) years). All patients and CSs were right-handed.

Based on the MRI findings, 8 patients had HS on the right and left side. Clinical and EEG findings were compatible with MRI. Demographic and clinical features of patients were summarized in Table 1.

None of the patients had dual pathology.

\section{SyMRI analysis results}

\section{Automated whole brain segmentation}

Left-sided HS patients (MY: $101.83 \pm 23.12$; WM: $373.36 \pm 58.87$ ) had significantly lower MY and WM volumes compared to CSs (MY: $132.55 \pm 14.62$; WM: $477.82 \pm 45.25)(p ; 0.003$ and 0.001 , respectively). Although there was a trend that right-sided HS patients had lower MY and WM volumes compared to CSs, it did not reach a significant difference (MY: 119.4 \pm 14.23; WM: 430.51 \pm 49.58 ) ( $p ; 0.073$ and 0.051 , respectively). GM, CSF, or NoN volumes did not show differences between patients and CSs.

\section{Quantification of SyMRI maps}

In patients with $\mathrm{HS}$, the MyFvol, MyV, and R1 values were significantly lower, and PD value was significantly higher in the ipsilateral temporal pole and sub-insular WM than contralateral regions for both observers $(<0.05$, Table 2 , Figs. 1 and 2). R2 value showed no difference.

The observers noticed that the expected fluorescent yellow/green color was turned into pale green in the areas with lower $\mathrm{MyV}$, especially in the ipsilateral temporal pole in HS (Fig. 1).

When compared to CSs, the MyV value was significantly decreased, and PD value was significantly increased in the ipsilateral side of temporal poles and temporal stems in both HS subgroups for both observers $(p<0.05$, Supplementary Table 1).

Left-sided HS patients showed lower mean MyV and $\mathrm{R} 1$ values and higher mean PD value in the ipsilateral posterior temporal lobe and sub-insular region in comparison
Fig.1 Coronal myelin maps derived from SyMRI of a 32-year-old male patient with right HS, suffering temporal lobe epilepsy for 4 years. Increased $\mathrm{T} 2$ signal intensity in the hippocampus, loss of internal structure of the hippocampus, and reduced hippocampal size are seen on T2WI (A, arrow). Although there was no apparent gray matter-white matter demarcation loss (B), prominent myelin reduction in the right temporal pole (coded as pale green $>$ yellow) compared to the contralateral side (bright fluorescent yellow $>$ green) is seen on colored myelin map ( $\mathbf{C}$, arrows). Luxol fast blue-H\&E stain of the white matter of the anterior temporal lobectomy section (D) shows myelin pallor easily observed by visual assessment compared to an autopsy control (E). The myelin fibers appear blue and neuropil appears pink
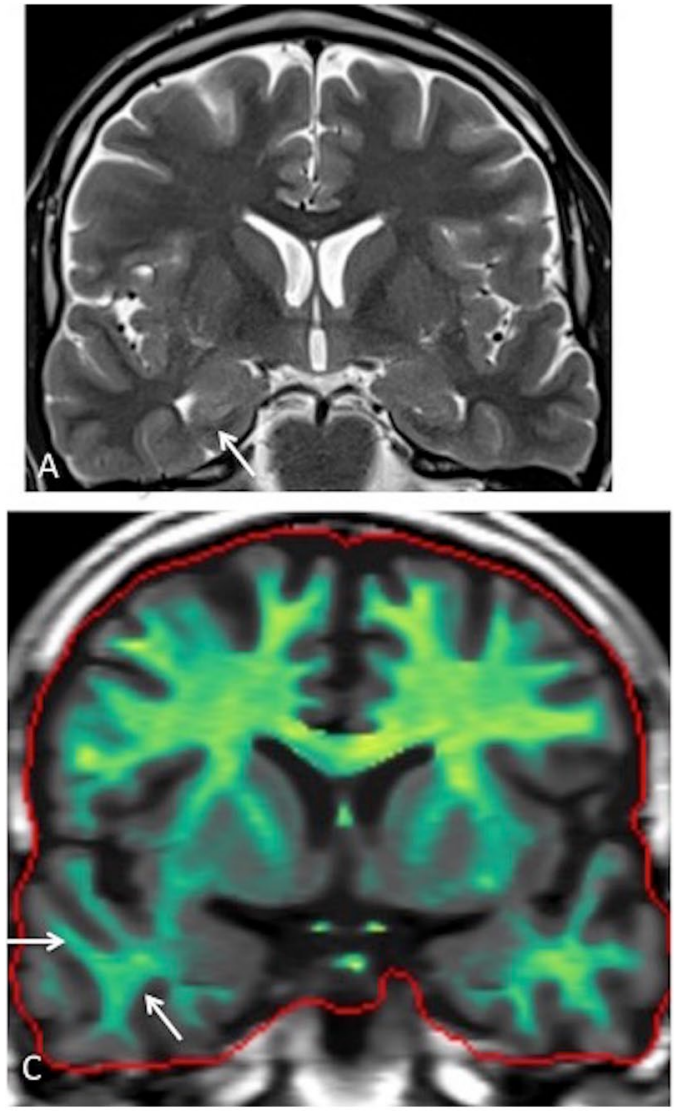
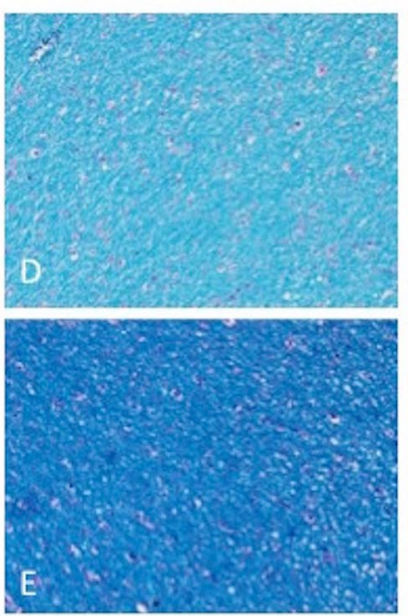
Table 1 Demographic and clinical features of patients with unilateral HS
Table 2 Myelin, R1, and PD values of statistically significant areas in patients with HS $(n=16)$

\begin{tabular}{llll}
\hline & HS $(n=16)$ & Right HS $(n=8)$ & Left HS $(n=8)$ \\
\hline Sex (F/M) & $6 / 10$ & $4 / 4$ & $2 / 6$ \\
Age (years) (min-max) & $32.5 \pm 11.3(19-48)$ & $32.75 \pm 7(19-42)$ & $32.3 \pm 13.4(21-48)$ \\
Onset of epilepsy (years) (min-max) & $14.6 \pm 7.7(1-39)$ & $14.8 \pm 10.7(1-28)$ & $14.5 \pm 26.1(2-39)$ \\
Duration of epilepsy (years) (min-max) & $17.8 \pm 3.5(4-32)$ & $17.8 \pm 17.6(4-29)$ & $17.8 \pm 12.7(5-32)$ \\
History of febrile convulsion & $8(50)$ & $5(62.5)$ & $3(37.5)$ \\
History of meningitis/encephalitis & None & None & None \\
History of trauma & $6(37.5)$ & $1(12.5)$ & $5(62.5)$ \\
History of or perinatal brain injury & $1(6.2)$ & None & $1(12.5)$ \\
Secondary generalized seizure & $9(56.2)$ & $5(62.5)$ & $4(50)$ \\
Status epilepticus & $1(6.2)$ & $1(12.5)$ & None \\
\hline
\end{tabular}

Values are expressed as mean $\pm \mathrm{SD}$ or $n(\%)$

\begin{tabular}{|c|c|c|c|c|c|c|c|}
\hline & & \multicolumn{2}{|l|}{ Temporal pole } & \multirow[t]{2}{*}{$p$ value $^{\mathrm{a}}$} & \multicolumn{2}{|l|}{ Sub-insular WM } & \multirow[t]{2}{*}{$p$ value $^{\mathrm{a}}$} \\
\hline & & Ipsilateral & Contralateral & & Ipsilateral & Contralateral & \\
\hline \multirow[t]{2}{*}{ MyFvol } & Ob1 & $0.042 \pm 0.007$ & $0.053 \pm 0.007$ & $p<.001>$ & $0.030 \pm 0.009$ & $0.038 \pm 0.010$ & $p=.002$ \\
\hline & $\mathrm{Ob} 2$ & $0.048 \pm 0.009$ & $0.057 \pm 0.012$ & $p<.001$ & $0.028 \pm 0.009$ & $0.058 \pm 0.091$ & $p=.003$ \\
\hline \multirow[t]{2}{*}{$\mathrm{MyV}$} & Ob1 & $20.437 \pm 3.516$ & $24.875 \pm 3.161$ & $p<.001$ & $14.825 \pm 3.500$ & $18.268 \pm 3.422$ & $p=.002$ \\
\hline & $\mathrm{Ob} 2$ & $20.844 \pm 2.571$ & $23.975 \pm 3.093$ & $p<.001$ & $13.594 \pm 4.283$ & $17.269 \pm 4.079$ & $p=.002$ \\
\hline \multirow[t]{2}{*}{ R1 } & Ob1 & $1.360 \pm 0.100$ & $1.479 \pm 0.117$ & $p<.001$ & $1.164 \pm 0.090$ & $1.221 \pm 0.0999$ & $p=.018$ \\
\hline & $\mathrm{Ob} 2$ & $1.349 \pm 0.095$ & $1.465 \pm 0.095$ & $p<.001$ & $1.138 \pm 0.095$ & $1.201 \pm 0.0973$ & $p=.007$ \\
\hline \multirow[t]{2}{*}{ PD } & Ob1 & $72.306 \pm 2.341$ & $69.393 \pm 2.071$ & $p<.001$ & $75.775 \pm 2.270$ & $73.5 \pm 2.209$ & $p=.002$ \\
\hline & $\mathrm{Ob} 2$ & $72.056 \pm 1.718$ & $69.931 \pm 2.057$ & $p<.001$ & $76.394 \pm 2.496$ & $74.119 \pm 2.767$ & $p=.002$ \\
\hline
\end{tabular}

$M y V$ myelin-correlated volume, $M y F v o l$ the mean amount of $\mathrm{MyV}$ in a single voxel, $R 1$ longitudinal relaxation rate, $P D$ proton density, $O b 1$ observer $1, O b 2$ observer 2

Values are expressed as mean $\pm \mathrm{SD}$

Units: MyV (ml), MyFvol (ml), PD (pu), R1 (s-1)

${ }^{a}$ Adjusted $p$ value was obtained using the Benjamini-Hochberg method to corresponding regions of the CSs for both observers $(p<0.05$, Supplementary Table 1$)$.

There was no statistical significance between the contralateral hemispheres of the HS subgroups and sidematched hemispheres of the CSs in any region.

Intra-class correlation for statistically significant results is given in Supplementary Table 2.

There was no significant difference in terms of WM and WMFvol in ipsilateral and contralateral hemispheric regions in HS patients or between patients and CSs.

Comparisons of hippocampi yielded lower GM, GMFvol, R1, and R2 values in both right and left HS patients than CSs $(p<0.05$, for both observers, ICC: $0.779-0.900$, Table 3 ). The PD value showed an increasing trend in patients without significance. No significant difference was found in the amygdala.

No significant correlation was found between quantitative values and none of the investigated clinical features $(p>0.05)$.

\section{Histopathological results}

Six patients (five right-sided HS and one left-sided HS) in this study underwent surgery consisting of hippocampectomy, amygdalectomy, and temporal lobectomy for refractory seizures $(n=6)$. Neuropathological evaluations revealed severe neuronal cell loss and gliosis predominantly in CA1 and CA4 regions. Luxol fast blue stain combined with $H \& E$ revealed pale blue staining in the WM, thus was interpreted as myelin pallor in the anterior temporal lobectomy sections (Fig. 1 B and C). No significant pathology was observed in the amygdala sections included in the specimen.

\section{Discussion}

This study has investigated the quantification in HS patients using SyMRI. With significantly lower MyFvol, MyV, and R1 and high PD values extensively in the temporal lobe WM and sub-insular regions in HS patients, alterations in 


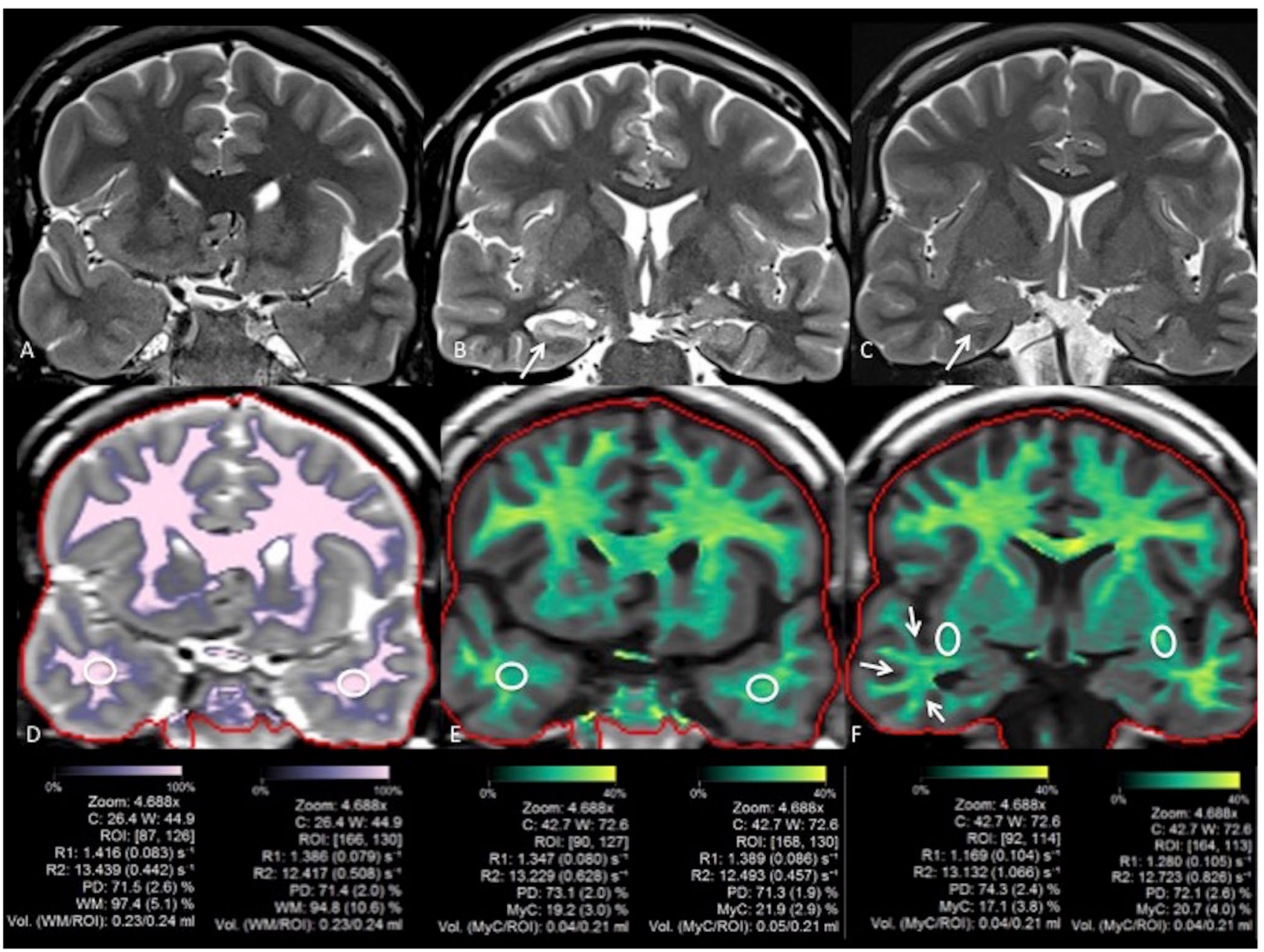

Fig.2 Consecutive coronal T2W images (A-C) of a 23-year-old male patient with right HS, suffering epilepsy for 15 years. Increased $\mathrm{T} 2$ signal intensity in the hippocampus, loss of internal structure of the hippocampus, and reduced hippocampal size are seen on $\mathrm{T} 2 \mathrm{~W}$ images $(\mathbf{B}, \mathbf{C})$. Coronal white matter $(\mathbf{D})$ and myelin maps $(\mathrm{E} \& \mathrm{~F})$ derived from SyMRI of same patient. The MyFvol, MyV, and R1 val- ues were decreased, and PD value was increased in the right temporal pole and sub-insular WM than contralateral regions on the myelin map without any color change in WM maps or any apparent graywhite matter demarcation loss. Myelin reduction in the right temporal lobe can easily be observed by visual assessment (arrows)

patients supporting SyMRI results. A semi-automated quantitative image analysis of myelin, as previously described in pathological specimens of a patient population of tuberous sclerosis, would reveal quantitative correlations with myelin assessment by SyMRI [24].

The validity of measurement of myelin by SyMRI was supported by previous studies. Quantitative results depicted the normal developmental pattern of myelination in pediatric patients in studies based on the normative quantitative segmentation of intracranial tissue [25] and acquired myelin partial volume values in different regions [26] on SyMRI. Also, measurement of myelin with SyMRI was correlated with Magnetization Transfer Saturation Index [27] and with postmortem brain studies using myelin sensitive luxol fast blue [28]. Underlying myelin and/or axonal abnormality of the WM by SyMRI was also shown in neurological diseases 
Table 3 Statistically significant results in comparison of hippocampi

\begin{tabular}{|c|c|c|c|c|c|c|c|}
\hline & & \multicolumn{2}{|c|}{ Right hippocampi } & \multirow[t]{2}{*}{$p$ value $^{\mathrm{a}}$} & \multicolumn{2}{|l|}{ Left hippocampi } & \multirow[t]{2}{*}{$p$ value $^{\mathrm{a}}$} \\
\hline & & R-HS & CSs & & L-HS & CSs & \\
\hline \multirow[t]{4}{*}{ Ob1 } & GM & $81.075 \pm 6.870$ & $95.010 \pm 2.210$ & $p=.002$ & $70.038 \pm 9.970$ & $95.170 \pm 2.030$ & $p=.001$ \\
\hline & GMFvol & $0.157 \pm 0.301$ & $0.205 \pm 0.029$ & $p=.005$ & $0.140 \pm 0.026$ & $0.204 \pm 0.302$ & $p=.001$ \\
\hline & $\mathrm{R} 1$ & $0.617 \pm 0.100$ & $0.782 \pm 0.033$ & $p=.002$ & $0.655 \pm 0.087$ & $0.804 \pm 0.061$ & $p=.001$ \\
\hline & $\mathrm{R} 2$ & $8.943 \pm 1.003$ & $10.732 \pm 0.427$ & $p=.002$ & $8.153 \pm 1.085$ & $10.732 \pm 0.545$ & $p=.001$ \\
\hline \multirow[t]{4}{*}{$\mathrm{Ob} 2$} & GM & $84.650 \pm 7.169$ & $98.87 \pm 0.733$ & $p=.005$ & $76.8 \pm 12.778$ & $98.08 \pm 1.320$ & $p=.004$ \\
\hline & GMFvol & $0.182 \pm 0.048$ & $0.196 \pm 0.015$ & $p=.014$ & $0.147 \pm 0.034$ & $0.196 \pm 0.015$ & $p=.002$ \\
\hline & $\mathrm{R} 1$ & $0.668 \pm 0.102$ & $0.809 \pm 0.038$ & $p=.006$ & $0.685 \pm 0.075$ & $0.821 \pm 0.026$ & $p=.001$ \\
\hline & $\mathrm{R} 2$ & $9.343 \pm 0.642$ & $10.869 \pm 0.260$ & $p=.002$ & $8.050 \pm 1.246$ & $10.623 \pm 0.432$ & $p=.001$ \\
\hline
\end{tabular}

$R / L H S$ right/left hippocampal sclerosis, $C S s$ control subjects, $G M$ gray matter, GMFvol the mean amount of GM in a single voxel, $R 1$ longitudinal relaxation rate, $R 2$ transverse relaxation rates, $O b 1$ observer 1 , Ob 2 observer 2

Values are expressed as mean $\pm \mathrm{SD}$

Units: GM (ml), GMFvol (ml), R1 (s-1), R2 (s-1)

${ }^{a}$ Adjusted $p$ value was obtained using the Benjamini-Hochberg method including demyelinating diseases, Sturge-Weber syndrome, and cognitive dysfunction in both normal and abnormal WM $[11,12,29-31]$.

The role in lateralizing epileptic focus and the impact of TLE on the organization of cerebral networks were assessed with quantitative measurements on DTI. In HS, widespread increased mean diffusivity (MD) and decreased fractional anisotropy (FA) more prominent in the ipsilateral hemisphere were reported in a meta-analysis study [32]. These changes showed variable alterations in terms of spatial pattern and degree in left and right HS patients across the studies, and it is not certain if right or left HS shows wider abnormality [33-36]. Additionally, Oguz et al. reported that decreased FA was more extensive in men with left HS and in women with right HS compared to sex-matched controls [37]. SyMRI findings representing myelin loss were more extensive in patients with left HS in our study. This may be related to the effect of patients' gender and male dominance in the left HS group.

Sclerosis of the hippocampus with gliosis and neuronal loss at various degrees is identified at histopathologic examinations of HS [38]. Our findings of decreased GM and GMFvol in the ipsilateral hippocampus with SyMRI reflect the neuronal loss observed in the histopathological specimens. However, in contradiction to previously reported changes frequently involving the amygdala in HS [39], we found no significant radiological and pathological abnormality in the amygdalae.

Neither we found a statistical abnormality in the thalami. Nonetheless, atrophy of medial dorsal thalamus and diffusion properties were shown by volumetric MRI [40] and DTI [41] studies, respectively, reflecting neuronal loss and gliosis in experimental [42], and postmortem histological studies in HS [43, 44]. Our failure of finding significant changes in the thalami using SyMRI may result from ROI placement method and the relatively small number of study populations.

Long-lasting pandemic conditions of COVID-19 apparently affected us as elsewhere in the world. Consequently, our study group and furthermore the number of patients undergoing temporal lobectomy were limited. Myelin alterations revealed by SyMRI in these patients also need to be investigated by other myelin imaging techniques on MRI especially on higher field scanners. One other limitation is that we could not assess all the structures belonging to the Papez circuit and other areas related to mesolimbic structures due to their small volumes. Despite normal brain MRI findings, subtle microscopic abnormalities cannot be completely excluded in subjects with a history of headache, vertigo, or peripheral facial paralysis, which constituted our control group.

\section{Conclusion}

SyMRI is a potential imaging tool for the investigation of myelin abnormality in patients with HS. This study has shown reduced myelin in the ipsilateral temporal lobe and sub-insular WM in HS patients with SyMRI. Whether this finding correlates with electrophysiological features and SyMRI could serve as lateralization of TLE need to be investigated.

Supplementary Information The online version contains supplementary material available at https://doi.org/10.1007/s00234-021-02824-6.

Acknowledgements We thank all our patients and healthy control subjects for their participation, the SyMRI team, MR technicians, and Ali Avci from Siemens Healthineers, Turkey, for their contribution in performing this study. 
Funding No funding was received for this study.

Data availability Restrictions apply to the availability of the data which were used under licence for the current study and thus are not publicly available.

Code availability This study was performed using special complimentary transitional licence by SyMRI and code availability is not applicable.

\section{Declarations}

Ethical approval Institutional review board approval was obtained (Hacettepe University Institutional Review Board).

Consent to participate Written informed consent for participation was obtained from all patients.

Consent for publication Written informed consent for publication was obtained from all patients including personal information or images of patients.

Conflict of interest None of the authors have a relevant conflict of interest.

\section{References}

1. Falconer MA, Serafetinides EA, Corsellis JA (1964) Etiology and pathogenesis of temporal lobe epilepsy. Arch Neurol 10:233-248. https://doi.org/10.1001/archneur.1964.00460150003001

2. Von Oertzen J, Urbach H, Jungbluth S et al (2002) Standard magnetic resonance imaging is inadequate for patients with refractory focal epilepsy. J Neurol Neurosurg Psychiatry 73:643-647. https:// doi.org/10.1136/jnnp.73.6.643

3. Lee DH, Gao FQ, Rogers JM et al (1998) MR in temporal lobe epilepsy: analysis with pathologic confirmation. AJNR Am J Neuroradiol 19:19-27

4. Meiners LC, van Gils A, Jansen GH et al (1994) Temporal lobe epilepsy: the various MR appearances of histologically proven mesial temporal sclerosis. AJNR Am J Neuroradiol 15:1547-1555

5. Achten E, Boon P, De Poorter J et al (1995) An MR protocol for presurgical evaluation of patients with complex partial seizures of temporal lobe origin. AJNR Am J Neuroradiol 16:1201-1213

6. Meiners LC, Witkamp TD, de Kort GA et al (1999) Relevance of temporal lobe white matter changes in hippocampal sclerosis. Magnetic resonance imaging and histology. Invest Radiol 34:38 45. https://doi.org/10.1097/00004424-199901000-00006

7. Camacho DL, Castillo M (2007) MR imaging of temporal lobe epilepsy. Semin Ultrasound CT MR 28:424-436. https://doi.org/ 10.1053/j.sult.2007.09.005

8. Carrete H Jr, Abdala N, Lin K et al (2007) Temporal pole signal abnormality on MR imaging in temporal lobe epilepsy with hippocampal sclerosis: a fluid-attenuated inversion-recovery study. Arq Neuropsiquiatr 65:553-560. https://doi.org/10.1590/s0004$282 \times 2007000400001$

9. Mitchell LA, Jackson GD, Kalnins RM et al (1999) Anterior temporal abnormality in temporal lobe epilepsy: a quantitative MRI and histopathologic study. Neurology 52:327-336. https://doi.org/ 10.1212/wnl.52.2.327

10. Andica C, Hagiwara A, Hori M et al (2018) Automated brain tissue and myelin volumetry based on quantitative MR imaging with various in-plane resolutions. J Neuroradiol 45:164-168. https:// doi.org/10.1016/j.neurad.2017.10.002

11. Park M, Moon Y, Han SH et al (2019) Myelin loss in white matter hyperintensities and normal-appearing white matter of cognitively impaired patients: a quantitative synthetic magnetic resonance imaging study. Eur Radiol 29:4914-4921. https://doi.org/10.1007/ s00330-018-5836-x

12. Andica C, Hagiwara A, Hori M et al (2019) Aberrant myelination in patients with Sturge-Weber syndrome analyzed using synthetic quantitative magnetic resonance imaging. Neuroradiology 61:1055-1066. https://doi.org/10.1007/s00234-019-02250-9

13. Granberg T, Uppman M, Hashim F et al (2016) Clinical feasibility of synthetic MRI in multiple sclerosis: a diagnostic and volumetric validation study. AJNR Am J Neuroradiol 37:1023-1029. https://doi.org/10.3174/ajnr.A4665

14. Hagiwara A, Hori M, Yokoyama K et al (2017) Synthetic MRI in the detection of multiple sclerosis plaques. AJNR Am J Neuroradiol 38:257-263. https://doi.org/10.3174/ajnr.A5012

15. Hagiwara A, Kamagata K, Shimoji K et al (2019) White matter abnormalities in multiple sclerosis evaluated by quantitative synthetic MRI, diffusion tensor imaging, and neurite orientation dispersion and density imaging. AJNR Am J Neuroradiol 40:1642-1648. https://doi.org/10.3978/j.issn.2223-4292.2015.02. 06

16. Hagiwara A, Hori M, Suzuki M et al (2016) Contrast-enhanced synthetic MRI for the detection of brain metastases. Acta Radiol Open 5:2058460115626757. https://doi.org/10.1177/2058460115 626757

17. Fujita S, Nakazawa M, Hagiwara A et al (2019) Estimation of gadolinium-based contrast agent concentration using quantitative synthetic MRI and its application to brain metastases: a feasibility study. Magn Reson Med Sci 18:260-264. https://doi.org/10.2463/ mrms.mp.2018-0119

18. Kang KM, Choi SH, Hwang M et al (2018) Application of synthetic mri for direct measurement of magnetic resonance relaxation time and tumor volume at multiple time points after contrast administration: preliminary results in patients with brain metastasis. Korean J Radiol 19:783-791. https://doi.org/10.3348/kjr. 2018.19.4.783

19. Warntjes M, Engstrom M, Tisell A et al (2016) Modeling the presence of myelin and edema in the brain based on multi-parametric quantitative MRI. Front Neurol 7:16. https://doi.org/10.3389/ fneur.2016.00016

20. Bobman SA, Riederer SJ, Lee JN et al (1985) Cerebral magnetic resonance image synthesis. AJNR Am J Neuroradiol 6:265-269

21. Riederer SJ, Suddarth SA, Bobman SA et al (1984) Automated MR image synthesis: feasibility studies. Radiology 153:203-206. https://doi.org/10.1148/radiology.153.1.6089265

22. Garbelli R, Milesi G, Medici V et al (2012) Blurring in patients with temporal lobe epilepsy: clinical, high-field imaging and ultrastructural study. Brain 135:2337-2349. https://doi.org/10.1093/ brain/aws 149

23. Ye Y, Xiong J, Hu J et al (2013) Altered hippocampal myelinated fiber integrity in a lithium-pilocarpine model of temporal lobe epilepsy: a histopathological and stereological investigation. Brain Res 1522:76-87. https://doi.org/10.1016/j.brainres.2013.05.026

24. Mühlebner A, van Scheppingen J, de Neef A et al (2020) Beyond white matter in tuberous sclerosis complex (TSC) cortical tubers. J Neuropathol Exp Neurol 79(10):1054-1064. https://doi.org/10. 1093/jnen/nlaa090

25. McAllister A, Leach J, West H et al (2017) Quantitative synthetic MRI in children: normative intracranial tissue segmentation values during development. AJNR Am J Neuroradiol 38:2364-2372. https://doi.org/10.3174/ajnr.A5398

26. Kim HG, Moon WJ, Han J et al (2017) Quantification of myelin in children using multiparametric quantitative MRI: a pilot 
study. Neuroradiology 59:1043-1051. https://doi.org/10.1007/ s00234-017-1889-9

27. Hagiwara A, Hori M, Kamagata K et al (2018) Myelin measurement: comparison between simultaneous tissue relaxometry, magnetization transfer saturation index, and $\mathrm{T} 1 \mathrm{w} / \mathrm{T} 2 \mathrm{w}$ ratio methods. Sci Rep 8:10554. https://doi.org/10.1038/s41598-018-28852-6

28. Warntjes JBM, Persson A, Berge J et al (2017) Myelin detection using rapid quantitative MR imaging correlated to macroscopically registered luxol fast blue-stained brain specimens. AJNR Am J Neuroradiol 38:1096-1102. https://doi.org/10.3174/ajnr.A5168

29. West J, Aalto A, Tisell A et al (2014) Normal appearing and diffusely abnormal white matter in patients with multiple sclerosis assessed with quantitative MR. PLoS ONE 9:e95161. https://doi. org/10.1371/journal.pone.0095161

30. Saccenti L, Hagiwara A, Andica C, et al. (2020) Myelin measurement using quantitative magnetic resonance imaging: a correlation study comparing various imaging techniques in patients with multiple sclerosis. Cells 9. https://doi.org/10.3390/cells9020393

31. Andica C, Hagiwara A, Nakazawa M et al (2016) The advantage of synthetic MRI for the visualization of early white matter change in an infant with Sturge-Weber syndrome. Magn Reson Med Sci 15:347-348. https://doi.org/10.2463/mrms.ci.2015-0164

32. Otte WM, van Eijsden P, Sander JW et al (2012) A meta-analysis of white matter changes in temporal lobe epilepsy as studied with diffusion tensor imaging. Epilepsia 53:659-667. https://doi.org/ 10.1111/j.1528-1167.2012.03426.x

33. Liu M, Concha L, Lebel C et al (2012) Mesial temporal sclerosis is linked with more widespread white matter changes in temporal lobe epilepsy. Neuroimage Clin 1:99-105. https://doi.org/10. 1016/j.nicl.2012.09.010

34. Focke NK, Yogarajah M, Bonelli SB et al (2008) Voxel-based diffusion tensor imaging in patients with mesial temporal lobe epilepsy and hippocampal sclerosis. Neuroimage 40:728-737. https://doi.org/10.1016/j.neuroimage.2007.12.031

35. Rodriguez-Cruces R, Concha L (2015) White matter in temporal lobe epilepsy: clinico-pathological correlates of water diffusion abnormalities. Quant Imaging Med Surg 5:264-278. https://doi. org/10.3978/j.issn.2223-4292.2015.02.06

36. Shon YM, Kim YI, Koo BB et al (2010) Group-specific regional white matter abnormality revealed in diffusion tensor imaging of medial temporal lobe epilepsy without hippocampal sclerosis.
Epilepsia 51:529-535. https://doi.org/10.1111/j.1528-1167.2009. 02327.x

37. Oguz KK, Tezer I, Sanverdi E et al (2013) Effect of patient sex on white matter alterations in unilateral medial temporal lobe epilepsy with hippocampal sclerosis assessed by diffusion tensor imaging. AJNR Am J Neuroradiol 34:1010-1015. https://doi.org/ 10.3174/ajnr.A3328

38. Thom M (2014) Review: Hippocampal sclerosis in epilepsy: a neuropathology review. Neuropathol Appl Neurobiol 40:520-543. https://doi.org/10.1111/nan.12150

39. Bote RP, Blazquez-Llorca L, Fernandez-Gil MA et al (2008) Hippocampal sclerosis: histopathology substrate and magnetic resonance imaging. Semin Ultrasound CT MR 29:2-14. https://doi. org/10.1053/j.sult.2007.11.005

40. Barron DS, Fox PM, Laird AR et al (2012) Thalamic medial dorsal nucleus atrophy in medial temporal lobe epilepsy: a VBM meta-analysis. Neuroimage Clin 2:25-32. https://doi.org/10. 1016/j.nicl.2012.11.004

41. Kim CH, Koo BB, Chung CK et al (2010) Thalamic changes in temporal lobe epilepsy with and without hippocampal sclerosis: a diffusion tensor imaging study. Epilepsy Res 90:21-27. https:// doi.org/10.1016/j.eplepsyres.2010.03.002

42. Bertram EH, Mangan PS, Zhang D et al (2001) The midline thalamus: alterations and a potential role in limbic epilepsy. Epilepsia 42:967-978. https://doi.org/10.1046/j.1528-1157.2001.04200 8967.x

43. Sinjab B, Martinian L, Sisodiya SM et al (2013) Regional thalamic neuropathology in patients with hippocampal sclerosis and epilepsy: a postmortem study. Epilepsia 54:2125-2133. https:// doi.org/10.1111/epi.12403

44. Margerison JH, Corsellis JA (1966) Epilepsy and the temporal lobes. A clinical, electroencephalographic and neuropathological study of the brain in epilepsy, with particular reference to the temporal lobes. Brain 89:499-530. https://doi.org/10.1093/brain/ 89.3.499

Publisher's note Springer Nature remains neutral with regard to jurisdictional claims in published maps and institutional affiliations. 